\title{
Estrategias para promover un ambiente académico respetuoso en el ámbito clínico
}

\author{
Strategies to promote a respectful academic environment in the clinical setting
}

\author{
Natalia Mejía ${ }^{1,2}$ Daniel Enrique Suárez
}

\section{Resumen}

El maltrato, la amenaza, el acoso, la discriminación y la violencia de género en la educación médica son fenómenos generalizados mundialmente y altamente prevalentes. Los comportamientos inapropiados hacia los estudiantes de medicina han sido reconocidos como una amenaza pues interfieren en la construcción de la identidad profesional, el bienestar estudiantil y éxito académico contribuyendo al agotamiento físico, el desgaste emocional, y el abuso de alcohol y otras sustancias psicoactivas. El impacto negativo de un ambiente jerárquico donde las relaciones de poder pueden tornarse abusivas cambia el enfoque empático y altruista que se espera de un profesional de la salud y perpetúa el maltrato por generaciones. En facultades de medicina de Latinoamérica, la prevalencia de conductas inapropiadas en estudiantes de pregrado y posgrado varía de 17 a 100\%, con una importante participación de profesores y residentes como perpetradores del maltrato. Es responsabilidad de los líderes en educación médica enfocar sus esfuerzos pedagógicos en formar profesionales humanizados, empáticos y felices en modelos de enseñanza que promuevan la dignidad y los derechos de los estudiantes. La profesión médica debe asumir un papel de liderazgo condenando las conductas inapropiadas y aportando un enfoque de tolerancia cero para todas las formas de intimidación y acoso en el lugar de aprendizaje y de trabajo, así como fomentar la igualdad de oportunidades en la fuerza laboral médica.

Palabras clave: prevención; educación médica; maltrato; acoso; discriminación; violencia.

\begin{abstract}
Harassment, threatening behaviours, discrimination, and gender-based violence in medical education are global and widespread phenomena. Inappropriate behaviours towards medical students have been recognised as a threat to medical education. They interfere with the construction of professional identity, student well-being, and academic success, contributing to physical exhaustion, emotional burnout, and alcohol abuse. The negative impact of a hierarchical environment where abusive power-drive relationships can modify the empathic and altruistic approach expected from a health professional as perpetuates abuse for generations. In Latin American medical schools, the prevalence of inappropriate behaviour in undergraduate and graduate students ranges from 17 to $100 \%$, with professors and residents as perpetrators of misconduct. Focus in medical schools must be based on training empathetic health professionals that embrace the rights of students and human dignity. The medical profession must take a leadership role in condemning inappropriate behaviour and bringing a zero-tolerance approach to all forms of bullying and harassment in hospitals and learning environments, as well as promoting equal opportunity in the medical workforce.
\end{abstract}

Keywords: prevention; harassment; medical education; bullying; violence; discrimination.

Fecha de envío: 2021-10-07 - Fecha de aceptación: 2021-12-06

\section{Introducción}

La construcción de la identidad profesional, el nivel de bienestar estudiantil y el éxito académico durante la carrera de medicina se basan en gran medida en el tipo y dinámica de las relaciones con otros miembros del equipo de asistencia en salud (pares, profesores, estudiantes en niveles avanzados de formación), los antecedentes socio-culturales del estudiante, la forma como se asumen las experiencias personales, así como en los modelos de identificación e ideales adquiridos durante los años de entrenamiento en los ecosistemas clínicos (Barragán, et al. 2021). Comportamientos 
inapropiados, tanto intencionales como involuntarios, hacia los estudiantes de medicina han sido reconocidos como una amenaza para la educación, pues interfieren en su proceso de aprendizaje y contribuyen al agotamiento físico, el desgaste emocional, y a la disminución en la percepción de bienestar. El impacto negativo de este ambiente sobre una identidad en formación cambia la mirada altruista que se espera de un profesional de la salud hacia personalidades menos empáticas, perpetuadoras del maltrato recibido en contra de pacientes, familias y compañeros, y refuerza la idea de que están peor que sus compañeros de otras profesiones y que habrían elegido una profesión diferente si hubieran sabido de antemano el alcance del maltrato que sufrirían (Sheehan et al., 1990). Incluso, Silver sugirió que las conductas inapropiadas en la formación médica eran percibidas erróneamente como una parte fundamental del camino para convertirse en médico y una forma de nutrir la resiliencia (Silver \& Glicken, 1990).

\section{Contexto mundial}

El maltrato, la amenaza, el acoso, la discriminación y la violencia de género en la educación médica son fenómenos mundiales y generalizados. Un meta-análisis que incluyó 51 estudios reporta que el $59,4 \%$ de los estudiantes de medicina en entrenamiento de pre y posgrado había experimentado al menos una forma de acoso o discriminación durante su carrera siendo el maltrato verbal la conducta mas prevalente $(68,8 \%)$ en el pregrado mientras que el tipo menos común fue el maltrato físico (9\%). Durante la formación en posgrados médico-quirúrgicos, la prevalencia de conductas inapropiadas se estimó en $63,4 \%$. Los residentes reportaron la discriminación de género como la forma más común de abuso $(66,6 \%)$, seguida del maltrato verbal (58,2\%). El tipo menos común, aunque en valores sorprendentemente altos, fue la discriminación racial (26,3\%). Para ambos niveles de formación, el maltratador fue un médico $(34,4 \%)$ seguido por pacientes y familias (21,9\%), residentes (15,6\%), enfermeros (15\%) y otros (3,1\%)(Nais et al., 2014).

Las respuestas al cuestionario de graduación de la Asociación americana de facultades de medicina (Association of American Medical Colleges Graduation Questionnaire 2021) reporta que poco se han modificado las conductas inapropiadas en los últimos 5 años. En 2017, haber sido avergonzado públicamente fue reportado por 129 residentes de un total de $14396(0,9 \%)$ mientras que para 2021, 154 estudiantes manifestaron esta queja de un total de 15460 (1\%). Las respuestas incluyeron cualquier comportamiento de profesores, enfermeras, residentes/pasantes, otros empleados o personal de la institución y otros estudiantes. No se tomaron en cuenta conductas realizadas por los pacientes. La misma encuesta determinó que, excluyendo la humillación en público por ser la mas prevalente, la sumatoria de otras conductas como maltrato o acoso físico, avances sexuales no invitados, favores sexuales en intercambio por notas, disminución o prelación de oportunidades de entrenamiento basadas en género, apodos ofensivos, así como menor nota o menos oportunidades de entrenamiento basada en género, raza o etnia, orientación sexual o creencias religiosas, fue informada por el 40,3\% de 15486 encuestados. A la pregunta de la persona causante de ese comportamiento, las respuestas porcentuales fueron: profesores en aula $(1,2 \%)$, profesores en rotaciones clínicas (14,1\%), residentes/internos (8,3\%), enfermeros $(3,8 \%)$, administradores $(0,6 \%)$, otros empleados del hospital $(1,6 \%)$ y estudiantes (1,4\%). La falta de confianza en el sistema educativo, el desconocimiento de los mecanismos de reporte en los hospitales y la normalización histórica de las conductas inapropiadas debilita la cultura de denuncia, perpetuando el ambiente maltratador. De 6190 respuestas, $72,7 \%$ de los estudiantes no reportaron el evento por ninguna vía institucional y las razones predominantes para no hacerlo fueron "porque no les pareció lo suficientemente grave para reportarlo" (53\%) o porque "no creían que se iban a tomar medidas al respecto" $(40,6 \%)$.

\section{Contexto latinoamericano}

El trasfondo cultural de Latinoamérica permea el ámbito clínico dando lugar a que comportamientos socialmente aceptados se tornen inadecuados en un ambiente jerárquico bajo estructuras de poder académicas o laborales. Existen características culturales relacionadas con el cuerpo, el género y la sexualidad que pueden borrar la línea entre un cortejo regular y un avance sexual no invitado. Manifestaciones de afecto como saludos de beso entre hombres, abrazos y besos en la calle o amamantar en espacios públicos hacen compleja la explicación de la interrelación entre hombres y mujeres en nuestro continente (Felliti \& Rizzot, 2016). Otra particularidad es la expectativa del rol del hombre dominante en la sociedad y la sumisión esperada de las mujeres (Mejía et al., 2018). Los estereotipos culturales y de género, el desequilibrio de poder inherente a la formación médica y, la naturaleza competitiva de la práctica y la formación han fomentado una cultura de intimidación y acoso que, con el tiempo, se ha generalizado e institucionalizado.

El ámbito académico propio de las facultades de medicina trasciende el aula y llega a ambientes clínicos y sociales cuya finalidad es ofrecer atención en salud. En los contextos clínicos, los estudiantes y los profesores pasan a ser un actor más de un vasto engranaje que incluye todo el talento humano en salud, los pacientes y sus familiares. En estas dinámicas, la jerarquía proporciona una cadena de mando en la que algunas personas son las responsables de tomar decisiones médicas complejas así el equipo bajo su mando hace efectivo el plan encaminado a salvar la vida o en 
general a prestar un mejor servicio. El maltrato en las escuelas de medicina latinoamericanas (Iglesias et al., 2005; Maida et al., 2006; Castrillón et al., 2010; Paredes et al., 2010; Bastías-Vega et al., 2011; Guevara-Cuellar et al.,2011; Silva-Villareal et al., 2013; Bermeo et al., 2015; Mejia et al.,2015; Millán et al.,2015; Munayco-Guillén et al., 2015; Peres et al., 2016; Fretes et al., 2016; Sánchez et al., 2016; Derive et al., 2017; Mariscal et al., 2017; Martínez \& Henríquez, 2018; Gali et al., 2019; Reyes-Arellano et al., 2019) parece tener la misma prevalencia que en reportes mundiales (tabla. 1) y las quejas mas frecuentes son de la misma índole como humillación, crítica excesiva en público, exclusión, menor acceso a la oportunidad de aprendizaje. En nuestra cultura se añaden otras dinámicas como el traspaso de responsabilidades, asignación de tareas que no corresponden a su nivel de entrenamiento y solicitud de llevar a cabo trámites administrativos ajenos a las competencias esperadas para el estudiante.

Tabla 1: Caracterización de conductas de maltrato, amenaza, acoso, discriminación y violencia de género en facultades de medicina latinoamericanas.

\begin{tabular}{|c|c|c|c|c|c|}
\hline País & Autor/Año & $\begin{array}{l}\text { Tamaño de muestra (n) } \\
\text { y tipo de estudiante }\end{array}$ & $\begin{array}{l}\text { Porcentaje de } \\
\text { reporte de conductas } \\
\text { inapropiadas }\end{array}$ & $\begin{array}{l}\text { Tipo y porcentaje de } \\
\text { conducta mas frecuente }\end{array}$ & $\begin{array}{l}\text { Tipo de Maltratador } \\
\text { mas frecuente }\end{array}$ \\
\hline \multirow{2}{*}{ Argentina } & (Mejía, 2005) & $\mathrm{n}=322$, Residentes & $89 \%$ & Crítica excesiva (75\%) & $\begin{array}{l}\text { Residente año superior } \\
(26 \%)\end{array}$ \\
\hline & (Galli, 2019) & $\mathrm{n}=183$ - Residentes & $100 \%$ & Maltrato verbal (21.3\%) & $\begin{array}{l}\text { Residente año superior } \\
(68 \%\end{array}$ \\
\hline Brasil & (Peres, 2016) & $\mathrm{n}=317$, Estudiantes de pregrado & $92.3 \%$ & $\begin{array}{l}\text { Maltrato recurrente } 30.1 \% \\
\text { Maltrato grave }(64.2 \%)\end{array}$ & N/A \\
\hline Bolivia & (Mariscal, 2007) & $\mathrm{n}=125$, Internos & $54.4 \%$ & Llamada de atención (69.6\%) & $\begin{array}{l}\text { Personal de enfermería } \\
(66.4 \%)\end{array}$ \\
\hline \multirow[b]{2}{*}{ Chile } & (Maida, 2006) & $\mathrm{n}=757$, Estudiantes de pregrado & $91 \%$ & Maltrato y humillación (41.6\%) & Profesores (66.4\%) \\
\hline & (Bastías - Vega, 2021) & $\mathrm{n}=264$, Estudiantes de pregrado & $98 \%$ & $\begin{array}{l}\text { Desmoralización y exigencias } \\
\text { desreguladas (35\%) }\end{array}$ & Profesores (N/A\%) \\
\hline \multirow{6}{*}{ Colombia } & (Paredes, 2010) & $\mathrm{n}=1500$, Estudiantes de pregrado & $19,68 \%$ & Humillación y acoso (27\%) & $\begin{array}{l}\text { Profesores }(9 \%) \\
\text { Compañeros (7\%) }\end{array}$ \\
\hline & (Castrillón, 2010) & $\mathrm{n}=210$, Estudiantes de pregrado & $75.2 \%$ & $\begin{array}{l}\text { Maltrato verbal }(60,5 \%) \\
\text { Crítica injustificada (60.4\%) }\end{array}$ & N/A \\
\hline & (Guevara-Cuellar, 2011) & $\mathrm{n}=128$, Estudiantes de pregrado & $40,6 \%$ & Maltrato psicológico (98\%) & $\begin{array}{l}\text { Profesores pre-clínicos } \\
25.9 \%\end{array}$ \\
\hline & (Millán, 2015) & $\mathrm{n}=375$, Estudiantes de pregrado & $18.7 \%$ & $\begin{array}{l}\text { Maltrato y amenaza verbal } \\
(14.9 \%)\end{array}$ & $(\mathrm{N} / \mathrm{A})$ \\
\hline & (Bermeo, 2015) & $\mathrm{n}=401$, Estudiantes de pregrado & $70,6 \%$ & Maltrato verbal (38.9\%) & Profesores (24.3\%) \\
\hline & (Sánchez, 2016) & $\mathrm{n}=89$, internos & $90,2 \%$ & $\begin{array}{l}\text { Maltrato y extralimitación de } \\
\text { funciones }(52.4 \%)\end{array}$ & $\begin{array}{l}\text { Residentes }(27.8 \%) \\
\text { Médicos }(20.2 \%)\end{array}$ \\
\hline Ecuador & (Martínez, 2018) & $\mathrm{n}=700$, Estudiantes de pregrado & $97 \%$ & $\begin{array}{l}\text { Humillación y maltrato verbal } \\
(90 \%)\end{array}$ & Profesores (70\%) \\
\hline \multirow{3}{*}{ México } & $\begin{array}{l}\text { (Iglesias-Benavides, } \\
\text { 2005) }\end{array}$ & $\mathrm{n}=404$, Estudiantes de pregrado & $30,9 \%$ & Maltrato psicológico (98\%) & $\begin{array}{l}\text { Profesores }(38.7 \%) \\
\text { Residentes }(38.7 \%)\end{array}$ \\
\hline & (Derive, 2017) & $\mathrm{n}=143$, Residentes & $84 \%$ & $\begin{array}{l}\text { Humillación y maltrato verbal } \\
(78 \%)\end{array}$ & $\begin{array}{l}\text { Residente del mismo } \\
\text { año }(90 \%) \\
\text { Personal de enfermería } \\
(78 \%)\end{array}$ \\
\hline & (Reyes-Arellano, 2019) & $\begin{array}{l}\mathrm{n}=2250 \\
\text { Estudiantes de pregrado }\end{array}$ & $27 \%$ & $\begin{array}{l}\text { Abuso de poder y autorita- } \\
\text { rismo }(80 \%)\end{array}$ & $\begin{array}{l}\text { Profesores (60\%) } \\
\text { Residentes (27\%) }\end{array}$ \\
\hline Panamá & (Silva-Villareal, 2013) & $\mathrm{n}=472$, Estudiantes de pregrado & $39,8 \%$ & Maltrato verbal (15\%) & N/A \\
\hline Perú & $\begin{array}{l}\text { (Munayco-Guillén, } \\
\text { 2015) }\end{array}$ & $\mathrm{n}=281$, Estudiantes de pregrado & $96.8 \%$ & Maltrato verbal $(89,8 \%)$ & $\begin{array}{l}\text { Profesores (84.3\%) } \\
\text { Estudiantes (32.4\%) }\end{array}$ \\
\hline Venezuela & (Freites, 2016) & $\mathrm{n}=120$, Residentes & $97.5 \%$ & Maltrato verbal (96.6\%) & $\begin{array}{l}\text { Residentes de año } \\
\text { superior }(78.6 \%)\end{array}$ \\
\hline
\end{tabular}




\section{Consecuencias físicas, psicológicas, académicas y emocionales del maltrato}

Es una situación generalizada y arraigada pero profundamente nociva, la percepción de que el auto sacrificio y la resiliencia son valores inherentes a la profesión médica. Para los estudiantes, el ingreso a la facultad de medicina genera unas expectativas erróneas de que "sin sacrificio no hay ganancia", y que se espera que, como médico, anteponga su profesión a su familia, a sus intereses personales y a su propia vida. Un estudio realizado en el 2017 en la facultad de medicina de la Universidad de los Andes que midió indicadores de bienestar y la salud mental de los estudiantes, determinó que la autoimposición de sacrificio, la carga académica y el ambiente de competitividad, son factores relacionados con pobre bienestar y síntomas de depresión en la población estudiantil (Suarez et al., 2020). Las fuentes de estrés en las facultades de medicina son ampliamente conocidas; el acceso restringido a los cupos, en especial a plazas de residencias médico-quirúrgicas, el costo elevado de las matrículas en universidades privadas, la asignación de tiempo personal a actividades asistenciales y académicas, el contacto con la enfermedad, la muerte y el sufrimiento humano desde años tempranos, hacen que la calidad de vida del estudiante de medicina exhiba alertas rojas (Rosenberg 1984, Guthrie et al.,1998, Costa et al., 2012). Estas alertas tienen correlación con un mayor abuso de alcohol y sustancias psicoactivas. Un estudio en estudiantes de medicina norteamericanos reporta que uno de tres cumple con los criterios diagnósticos de abuso de licor, doblando en frecuencia a estudiantes de otras facultades (Jackson et al., 2016). Otro estudio en estudiantes de medicina de una universidad privada latinoamericana, evaluó en 559 sujetos mediante un formulario de autoreporte, la prevalencia de consumo de sustancias psicoactivas. La prevalencia, si bien menor a la descrita en Norteamérica, se reporta en 15,1\% para alcohol, entre 18,5 - 57,2\% para marihuana y $0,5-2,8 \%$ para cocaína (Calletti et al., 2021). El consumo de alcohol y otras sustancias se refuerza dentro del ciclo destructivo de depresión, falta de autoestima y sensación de no ser suficiente (Owens, 2018). Si a los estresores habituales de la carrera se adicionan otras causas de ansiedad como conductas intimidantes, dinámicas abusivas de poder, violencia verbal y humillación, las víctimas reportarán dificultades en la concentración y menor satisfacción con la elección de la carrera y, por consiguiente, un mayor índice de deserción (Timm, 2014).

El patrón de relaciones interpersonales abusivas en medicina, no solo se perpetúa, sino que, se incrementa en el tiempo (Peres et al., 2016). La jerarquía hospitalaria pone en riesgo de maltrato a quienes están en la base de la pirámide de subordinación. Ésta dinámica se vive, se aprende, se interioriza y finalmente se aplica a los nuevos subordinados, con la premisa de"si antes me trataron mal, ahora estoy en posición de tratar mal a otros" en una suerte de herencia cíclica tóxica o legado transgeneracional (Rautio et al., 2005). El orden moral preestablecido en las facultades de medicina, donde el currículo oculto apoya una cultura de vida centrada en el estudio y el sufrimiento, crea una atmósfera que influye tácita pero nocivamente en el comportamiento profesor-alumno y alumno-paciente (Becher, 1989). Sheehan y colaboradores encontraron que los estudiantes que fueron acosados frecuentemente tenían menos probabilidades de completar las tareas o brindar una atención óptima al paciente, además de exhibir mayores problemas de salud emocional y trastornos de la vida familiar en comparación con los estudiantes no acosados (Sheehan et al., 1990).

El acoso sexual es mas frecuente en la educación médica que en otras facultades y es experimentado de forma desproporcionada por estudiantes femeninas. En la jerarquía administrativa y operativa de un hospital, el acoso sexual puede facilitarse en una relación de poder que el acosador cree poseer en relación con la víctima, derivado del estatus formal en el lugar de trabajo (Rautio et al., 2005). Además, la medicina es una carrera inmersiva, el trabajo clínico se caracteriza por rupturas estructuradas en las barreras a la intimidad con requisitos (como los turnos nocturnos) que hacen que las líneas divisorias entre el trabajo y la vida social se difuminen. Los médicos acosados por otros médicos constituyen una población invisible de víctimas, siendo más prevalente en organizaciones que actúan como guardianas del avance profesional como hospitales universitarios y en entornos donde los hombres superan en número a las mujeres. El discurso social en torno al acoso sexual como la invalidación de la experiencia de la víctima, la auto-culpa, la minimización de la situación, terminan perpetuándolo (Stone et al., 2019).

\section{Contexto Universidad de los Andes}

El programa de desarrollo integral (PDI) 2021 - 2025 de la Universidad de los Andes define tres objetivos preponderantes, así como un conjunto de iniciativas, reformas, inversiones y metas concretas, en respuesta a una coyuntura mundial excepcional enmarcada por la pandemia de la COVID-19 así como por un conjunto de transformaciones de largo plazo, ambientales, tecnológicas, sociales, políticas y demográficas. En ese optimismo transformador el PDI plantea como su segundo sueño Una universidad que impulse el bienestar de todos sus integrantes, el pluralismo, la equidad de género y la diversidad. Dos de los pilares que apoyan la consecución de esa meta es fomentar un ambiente respetuoso de la diversidad, los diferentes experimentos de vida y las diferentes visiones de la sociedad, así como la promoción del bienestar y la salud mental y física de estudiantes, profesores y trabajadores administrativos. La iniciativa gira en torno a la idea del bienestar como responsabilidad de toda la comunidad uniandina. Hace énfasis en el fortalecimiento 
de la resiliencia y la capacidad de adaptación de los jóvenes, con modelos de intervención flexibles y viables que superen el impacto de la consejería individual. Se formará a los profesores y equipos administrativos en una cultura empática con los problemas de salud mental y, se protegerá el proyecto académico de los estudiantes con este tipo de dificultades. Existe una relación casi simbiótica entre el bienestar y el éxito estudiantil. Y este, es responsabilidad de todos, los mismos estudiantes, los profesores, las unidades administrativas de servicios y las unidades de soporte (Plan de desarrollo integral, Universidad de los Andes, 2021).

Desde su creación, la Universidad de los Andes ha buscado ser un espacio seguro y garantizar la dignidad de toda la comunidad académica. En este sentido, en el año 2016 la universidad promulga el protocolo MAAD (siglas de maltrato, acoso, amenaza, discriminación y violencia de género orientado a la prevención y hacer un abordaje efectivo de estos casos. Con este recurso, liderado por la Ombudsperson, los estudiantes, profesores y administrativos, pueden poner en conocimiento de la universidad aquellos casos en los que consideren que han recibido algún tipo de agresión por otros miembros de la comunidad que perturbe la convivencia y la tranquilidad universitaria, o lesione el buen nombre de la universidad; bien sea que la agresión haya ocurrido en el campus o fuera de él, incluyendo el uso de las plataformas tecnológicas. Este trabajo está apoyado por la labor de los consejeros MAAD: profesores y administrativos que se han postulado voluntariamente para ofrecer espacios de escucha, orientación y prevención que promuevan el cuidado constante de los otros. Otras dependencias, como la decanatura de estudiantes, apoyan emocionalmente a los estudiantes víctimas de conductas MAAD.

Otra iniciativa universitaria que fomenta el bienestar y educa a la comunidad en la identificación y denuncia de conductas MAAD es el grupo no es normal, campaña de la facultad de derecho que abre espacio de discusión sobre los acontecimientos de inequidad de género, sexismo y acoso sexual que puedan ocurrir en la Universidad de los Andes.

\section{Glosario MAAD en la Universidad de los Andes}

- Acoso: Todo comportamiento reiterado que, por acción u omisión, tiene el propósito o produce el efecto de atentar contra la dignidad de una persona, a la vez que crea un entorno en el que prima la intimidación, la indefensión y la humillación.

- Acoso sexual: Cualquier insinuación, solicitud o conducta sexual indeseada que puede crear un ambiente hostil, intimidatorio y ofensivo. Por tanto, toda conducta de carácter sexual que hace que una persona se sienta incómoda es acoso sexual. Toda persona puede ser víctima de acoso sexual sin importar su género.
- Amenaza: Todo acto en el que una persona, por cualquier medio, atemorice o dé a entender que quiere causar algún mal a otra persona, familia, comunidad o institución, con el propósito de causar zozobra, temor o terror.

- Discriminación: Cualquier acto, política o expresión verbal que implique el trato inequitativo, injusto o denigrante de una persona a partir de una característica personal como: edad, raza, sexo, género, orientación sexual, nacionalidad, religión, creencia, postura política, condición de discapacidad, etc.

- Maltrato: Cualquier acto de violencia contra la integridad física o moral, la libertad física sexual o los bienes de una persona. Cualquier comportamiento que menoscabe la autoestima y la dignidad o cualquier expresión verbal injuriosa o ultrajante que lesione la integridad o los derechos fundamentales de la persona.

- Violencia de género: Todo acto de violencia, basado en el género o la preferencia sexual, que tenga o pueda tener como resultado un daño o sufrimiento físico, sexual o psicológico.

\section{Estrategias de la facultad de medicina para promover el bienestar y una convivencia segura}

Si bien nuestra facultad se ha construido sobre una sólida base de principios éticos y profesionales, hemos percibido que la educación médica debe hacer esfuerzos extras para erradicar diferentes formas de maltrato que siguen estando presentes en nuestros ámbitos de formación. En este sentido, desde 2019 la facultad de medicina, así como el hospital universitario de la Fundación Santa Fe han trabajado sinérgicamente para identificar y caracterizar conductas inapropiadas en ámbitos clínicos enmarcadas dentro de conductas MAAD, y así generar, promover y promocionar una cultura de respeto reiterando una posición de cero tolerancia ante este tipo de comportamientos.

El trabajo conjunto se desglosa en 4 estrategias, algunas de ellas finalizadas, otras en curso y otras previstas para ser aplicadas en 2022:

\section{Estrategias de medición y caracterización del problema}

- $\quad$ Encuesta Fundación Santa Fe de Bogotá (FSFB): liderada por la dirección general de la FSFB y aplicada el segundo semestre de 2020 a todos los funcionarios administrativos y del talento humano en salud, incluidos los estudiantes de pregrado y posgrado de la Universidad de los Andes y de otras universidades. La encuesta denominada "El respeto y la inclusión son el camino" buscó entender estas dinámicas de relacionamiento entre los diferentes roles de las personas que 
- hacen parte del hospital universitario al mismo tiempo que midió la ocurrencia e intensidad de las conductas inapropiadas en este ámbito para dirigir más objetivamente las estrategias de promoción de un ambiente de sana convivencia.

- Encuesta de salud mental en estudiantes de medicina: este trabajo liderado por profesores de la facultad de medicina de la Universidad de los Andes en 2017, permitió identificar los factores determinantes de ansiedad y depresión, así como caracterizó otras conductas de riesgo asociadas a detrimento de la salud mental (Suárez et al., 2016).

- Encuesta percepción de conductas inapropiadas en la facultad de medicina: liderada por la decanatura de medicina con el apoyo del departamento de psicología. La intervención en grupos focales se está aplicando en el segundo semestre de 2021 y pretende medir en las diferentes poblaciones de nuestra facultad, el impacto de las estrategias conjuntas para promover el cambio de cultura hacia una zona de respeto e inclusión en el ámbito clínico.

\section{Estrategias de Educación, visibilidad y sensibilización}

- Construcción de un manifiesto interinstitucional (Uniandes-FSFB) de tolerancia cero a conductas MAAD: liderado por el consejo directivo de la universidad y la junta directiva de la FSFB. Se hizo público en el aniversario del hospital universitario en abril de 2021, comprometiendo todos los esfuerzos en asegurar un ambiente en que todos los seres humanos se sientan acogidos y respetados y encuentren condiciones propicias para la plena expresión de su personalidad y el alcance de su potencial.

- Seminarios educativos: De septiembre 2020 a marzo 2021 se han realizado siete eventos en modalidad remota en un esfuerzo conjunto del comité de género de la facultad de medicina y la Fundación Santa Fe a través del eje de educación y gestión del conocimiento. Estas reuniones pretenden educar a la comunidad académica y hospitalaria sobre perspectiva de género, inclusión, asimetría en el relacionamiento hospitalario, micromachismos en medicina, entre otros temas. Han contado con la participación de directivos de empresas de salud, de líderes de opinión, expertos en género, profesores de otras facultades y de otras universidades, y son moderadas por estudiantes. La grabación de los eventos pueden ser consultados (Univerdidad de Los Andes Colombia, 2021).

- Talleres prácticos en MAAD para profesores: estrategia educativa liderada por la facultad de medicina con el apoyo del Comité MAAD y el centro de ética aplicada de la Universidad de los Andes. Contó con la participación de 162 profesores de la facultad de medicina y estuvo orientada a la sensibilización, la identificación y la prevención de conductas inapropiadas en el ámbito clínico.

- Talleres en MAAD para estudiantes: estrategias educativas obligatorias y continuas lideradas por la facultad de medicina y sus consejeros MAAD, aplicada en semestres considerados de riesgo para ansiedad y burnout.

- Promoción de las líneas de denuncia: la facultad de medicina, los consejeros MAAD y el comité MAAD con el apoyo de los estudiantes del consejo estudiantil interno de medicina (CEIM) dan a conocer a través de diferentes medios (correos, página web, redes sociales, profesores consejeros) las líneas de denuncia institucionales.

- Campaña social media \#YoRespetoTuRespetas y \#MDiversa: se han incentivado campañas en Twitter e Instagram de actitudes responsables y respetuosas como usuarios de medios sociales y como parte de una misma comunidad. Esta iniciativa parte del comité disciplinario de la facultad de medicina y busca la sensibilización ante la responsabilidad que deben tener los usuarios de las redes sociales cuando hablamos de otros seres humanos. Con esta línea permanente de sensibilización se busca difundir que somos una facultad diversa y respetuosa con la diferencia.

- Taller atención en salud a pacientes con identidad de género diversa: liderado por el comité de género de la facultad de medicina utilizó la simulación para capacitar a estudiantes en la atención de pacientes con diversidad de género.

- Participación de estudiantes en Fundación al Día: la dirección general de la Fundación Santa Fe incluyó a los representantes estudiantiles del consejo estudiantil interno de medicina (CEIM), del consejo académico y el consejo superior a la reunión semanal administrativa del hospital universitario para hacerlos participe de las decisiones institucionales que en ella se tomen.

\section{Estrategias de Organización y Vigilancia}

- Creación del comité de bienestar de la facultad de medicina: en enero de 2020 se creó un comité con miembros plurales de la facultad de medicina. Se reúne quincenalmente y fomentan la salud física, mental y emocional de nuestra comunidad a través de diferentes estrategias que van desde reforzar la consejería individual, grupos focales con énfasis en el autocuidado, creación de espacios diferentes a los académicos para proveer esparcimiento, hasta una revisión exhaustiva de la "carga" académica dentro de la reforma curricular de pregrado. 
- En las demás instituciones diferentes a nuestro hospital base donde cursan prácticas clínicas nuestros estudiantes y residentes, se firma un compromiso bilateral de cero tolerencia a conductas inapropiadas en el ámbito clínico. En algunos centros asistenciales se incluyeron políticas institucionales de respeto y tolerancia en la inducción de todo el personal nuevo que ingresa a ellas.

- Designación de 3 consejeros/as MAAD para la facultad de medicina.

- Párrafo introductorio consignado en el estatuto profesoral de medicina que refuerza la actividad docente enmarcada en deberes de ética y respeto.

- Creación y divulgación del manual para fortalecimiento de la cultura Fundación Santa Fe de Bogotá.

- Creación del comité de género de la facultad de medicina: nace en octubre de 2020, cuenta con la participación de estudiantes, profesores y médicos institucionales del hospital universitario. con reuniones semanales, apoya iniciativas de sensibilización sobre violencia de género y participa en estrategias de educación continua con otros hospitales en convenio.

- Creación de la figura estudiantil denominada PAAR (promotores de ambiente académico respetuoso): prevista para 2022, permitirá que los estudiantes identifiquen situaciones MAAD en los diferentes ámbitos clínicos y promuevan el respeto mas allá de las aulas.

- Cambio en la designación de las funciones del consejo de facultad para que los casos de conductas inapropiadas sean tratados en ese ámbito.

- Designación de un psicólogo de decanatura de estudiantes para atención exclusiva, dos veces por semana, a los estudiantes de medicina en el centro de prácticas de la facultad.

\section{Estrategias y tratamiento específico denuncias anónimas y denuncias formales}

- Las denuncias que han sido recibidas tanto a la facultad como en la Fundación Santa Fe han tenido el curso establecido través de los órganos competentes para tal fin en cada una de las dos instituciones, privilegiando la confidencialidad y el debido proceso para ambas partes. Algunas han derivado en la desvinculación del profesor, otras han derivado en procesos disciplinarios en el Comité MAAD o en el comité de ética y credenciales de la FSFB.

\section{Conclusión}

La facultad de medicina inició un trabajo dirigido a mejorar los factores generadores de ansiedad, entre ellos las conductas MAAD, apoyada en la decanatura de estudiantes, el CEIM y la omdbusperson. Ha enfocado sus esfuerzos pedagógicos en formar profesionales humanizados a través de dos cursos de habilidades de comunicación, y así mismo ha ido capacitando a los profesores en trabajar situaciones que no repliquen modelos de enseñanza que vulneren la dignidad y los derechos de los estudiantes. La profesión médica debe asumir un papel de liderazgo al condenar la amenaza y el acoso, la discriminación y la violencia de género aportando un enfoque de tolerancia cero para todas las formas de intimidación y acoso en el lugar de aprendizaje y de trabajo, así como fomentar la igualdad de oportunidades en la fuerza laboral médica.

Los resultados de percepción de maltrato en las facultades de medicina de todo el mundo enfatizan en la necesidad de desarrollar y mantener una atmósfera de crecimiento académico, imparcial y sana que permita el desarrollo de las habilidades médicas y el constructo del perfil profesional necesario para el buen ejercicio de la medicina. La intervención pedagógica en el personal docente para influir en comportamientos y hábitos arraigados, así como la revisión del currículo oculto en términos de orden moral, se hace necesaria.

\section{Reconocimientos}

Agradecemos a la dirección general de la Fundación Santa Fe de Bogotá por aportar información relevante para el artículo.

Mejía N contribuyó con la escritura del artículo. Suárez D contribuyó con la recopilación de la bibliografía y la revisión final del documento.

No hay conflictos de interés declarados por los autores.

\section{Referencias}

Bastias N, Fasce E, Ortiz L, Pérez C. \& Schaufele P. (2011). Bullying y acoso en la formación médica de posgrado. Rev. Educ. Cienc. Salud 8,45-51.

Barragán OL, Avalos ML. \& Palos MU. (2021). La identidad profesional médica: una revisión narrativa. Caleidoscopio - Revista semestral de ciencias sociales y humanidades 44.

Becher T. (1989). Academic tribes and territories: Intellectual enquiry and the cul- tures of disciplines Stony Stratford: Society for Research into Higher Education \& Open University Press.

Bermeo JL et al. (2016). Abuso académico a estudiantes de pregrado por parte de docentes de los programas de Medicina de Manizales. Colombia Revista de la Facultad de Medicina 64. 
Calletti Godoy MJ, Elsaca Merino E, Kobus Garán, VP. \& Santander J. (2021). Consumo de alcohol, marihuana y cocaína en estudiantes de medicina de la Pontificia Universidad Católica de Chile. ARS MEDICA Revista de Ciencias Médicas 46, 11-16.

Castrillón JJ, Cerón AC, . Collazos AK, Molina AM, Osorio J, Ospina A, Páez, ML, Rico D \& Zamrano OE. (2012). Factores que inciden en la motivación académica en un programa de medicina, Manizales, Colombia. Archivos de Medicina (Col) 12, 46-61.

Costa EF, Santos SA, Santos AT, Melo EV, Andrade TM. (2012) Burnout Syndrome and associated factors among medical students: a cross- sectional study. Clinics (Sao Paulo) 67, 573-580.

Derive, Setal. (2018) Percepción de maltrato durante la residencia médica en México: medición y análisis bioético. Investigación educ. médica 7.

Felitti K. \& Rizzotti A. (2016). El “machismo latinoamericano" y sus derivas en la educación internacional: reflexiones de estudiantes estadounidenses en Buenos Aires. Magis, Revista Internacional de Investigación en Educación 9, 13-28.

Freites Hernández ML, Duque DM, Fernández R \& González Blanco M. (2016). Violencia laboral en el Programa de Especialización en Obstetricia y Ginecología: percepción del residente. Revista de Obstetricia y Ginecología de Venezuela 76, 85-92.

Galli A, Gimeno G, Lobianco MD, Swieszkowski S, Grancelli H, Kazelian L, Lapresa S, Pagés M. \& Duronto E. (2020). Maltrato en la formación médica: situación en las residencias de cardiología. Revista Argentina de Cardiología 88, 48-54.

Guevara-Cuéllar CA, Botero-Restrepo S, Borrero-Castro IL. \& Sinisterra J. (2011). Prevalencia de abuso y factores asociados en una escuela de medicina colombiana. Colombia Médica 42.

Guthrie E, Black D, Bagalkote H, et al. (1998). Psychological stress and burnout in medical students: a five-year prospective longitudinal study. J R Soc Med 91, 237-243.

Iglesias Benavides. (2005). Maltrato del estudiante de medicina. Percepción de 404 alumnos de cuarto, quinto y sexto años de la carrera. Med Univer 7, 191-202.

Jackson ER, Tait S, Hasan O, Satele DV. \& Dyrbye LN. (2016). Burnout and Alcohol Abuse/Dependence Among U.S. Medical Students, Academic Medicine 91,1251-1256.

Maida SAM, Herskovic MV, Pereira SA, Salinas-Fernández L. \& Esquivel CC. (2006). Perception of abuse among medical students of the University of Chile. Rev Med Chil 134, 1516-1523.
Mariscal E, Navia O, Paniagua, J, de Urioste N, Rodrigo. \& Espejo EE. (2007). Maltrato y/o violencia: fenómeno de estudio en centros de enseñanza asistenciales: Internado Rotatorio de Medicina gestión 2005 - 2006. Cuadernos Hospital de Clínicas 52, 46-54.

Martinez. (2018). El 19. 97\% de estudiantes de Medicina en Ecuador son víctimas de maltrato durante su formación. Accedido en: https://www.edicionmedica.ec/secciones/profesionales/97-de-estudiantes-de-medicina-en-ecuador-son-v-ctimas-de-maltrato-durante-su-formaci-n-91938 el 13 de octubre de 2021.

Medical School Graduation Questionnaire 2021 all schools summary report. Accedido en: https://www.aamc.org/media/55736/ download el 10 de octubre de 2021.

Mejía C, Pulido-Flores J, Quiñones-Laveriano D, Nieto-Gutierrez W. \& Heredia P. (2018). Machismo entre los estudiantes de medicina peruanos: Factores socio-educativos relacionados en 12 universidades peruanas. Revista Colombiana de Psiquiatría $4 \mathbf{8}$.

Mejía R, Diego A, Alemán M, Maliandi MR. \& Lasala F. (2005). Perception of mistreatment during medical residency training. Medicina 65, 295-301.

Millan Reyes LC, Barrera Sanchez LF \& Ospina Diaz JM. (2015). Caracterización del bullying en estudiantes de medicina de Tunja, Boyacá. Revista Virtual Universidad Católica del Norte 45, 101-112.

Munayco-Guillén F, Cámara-Reyes A, Muñoz-Tafur LJ, Arroyo-Hernández H, Mejia CR, Lem-Arce F. \& Miranda-Soberón UE. (2008). Características del maltrato hacia estudiantes de medicina de una universidad pública del Peru. Rev Peru Med Exp Salud Publica 33, 58-66.

Naif F, Soobiah C, Hong Chen M, Lillie E, Perrier P, Tashkhandi M, Straus SE, Mamdani M, Al-Omran M. \& Tricco AC. (2014). Harassment and discrimination in medical training: a systematic review and meta-analysis. Review Acad Med 89,817-27.

Owens B. (2018). Growing concern over medical students' excessive drinking. Canadian Medical Association Journal 190, 215.

Paredes OL, Sanabria-Ferrand PA, González-Quevedo LA, \& Moreno-Realphe SP. (2010) "Bullying" en las facultades de medicina colombianas, mito o realidad. Rev. Med 18,161-72.

Peres MF, Babler F, Arakaki JN, Quaresma IY, Barreto AD, Silva AT, Eluf-Neto J. (2016). Mistreatment in an academic setting and medical students' perceptions about their course in São Paulo, Brazil: a cross-sectional study. Sao Paulo Med J 134, 130-137. 
Rautio A, Sunnari V, Nuutinen M. et al. (2005). Mistreatment of university students most common during medical studies. BMC Med Educ 5, 36.

Reyes AWA, Ramírez MT, Ponce de León ME \& García JJ. (2019). Maltrato en el pregrado de la Facultad de Medicina de la UNAM. Inv EdMed 8.

Rosenberg DA. Medical student abuse (1984). An unnecessary and preventable cause of stress. JAMA 251, 739-742.

Sánchez N, Bonilla L, Rodríguez M, Sandoval G, Alzate JP, Murcia N, Suárez, M, Luque S, Arteaga JM, Galván J \& Eslava-Schmalbach J. (2016). Frequency of bullying perceived in clinical practices of last year interns of a medicine school: Cross sectional study. Revista de la Facultad de Medicina 64, 447.

Sheehan KH, Sheehan DV, White K, Leibowitz A \& Baldwin DC. (1990). A Pilot Study of Medical Student 'Abuse': Student Perceptions of Mistreatment and Misconduct in Medical School. JAMA 263, 533-537.

Silva-Villarreal S, Castillo S, Eskildsen E, Vidal P, Mitre J, \& Quintero J. (2013). Prevalencia de bullying en estudiantes de los ciclos básicos y preclínicos de la carrera de medicina de la Universidad de Panamá. Arch. Med 9,1-8.
Silver HK \& Glicken AD (1990). Medical student abuse, Incidence, Severity and Significance. JAMA 263, 527-32.

Stone L, Phillips C, \& Douglas KA. (2019). Sexual assault and harassment of doctors, by doctors: a qualitative study. Med Educ 53, 833-843.

Suárez DE , Cardozo AC , Villarreal ME \& Trujillo EM. (2020). Non-Heterosexual Medical Students Are Critically Vulnerable to Mental Health Risks: The Need to Account for Sexual Diversity in Wellness Initiatives. Teaching and Learning in Medicine 33, 1-9.

Timm A. (2014).'It would not be tolerated in any other profession except medicine': survey reporting on undergraduates' exposure to bullying and harassment in their first placement year. British Medical Journal MJ Open 4,e005140.

Univerdidad de Los Andes Colombia. (2021). Comité de género Facultad de Medicina. Accedido en: https://medicina.uniandes. edu.co/comite-de-genero el 15 octubre de 2021. 